\title{
Erratum: Robust linear coupling correction with $N$-turn maps [Phys. Rev. ST Accel. Beams 6, 062801 (2003)]
}

\author{
Wolfram Fischer \\ (Received 23 August 2007; published 31 August 2007)
}

DOI: $10.1103 /$ PhysRevSTAB.10.089901

PACS numbers: 29.20.Dh, 29.27.Ac, 29.27.Bd, 99.10.Cd

This erratum discusses the problem of small denominators in Eqs. (8) and (9) in the original paper [1], where it was not treated correctly. The change in this discussion should not have any implications for other parts of the paper. The text from after Eq. (6) until after Eq. (11) should be replaced with the following: [2]

The underlined matrices denote one-turn maps in the eigenbasis. The matrix $\mathbf{G}$ and its inverse $\mathbf{G}^{-1}$ can be expressed as

$$
\mathbf{G}=\left(\begin{array}{cc}
g \mathbf{I} & -\overline{\mathbf{R}} \\
\mathbf{R} & g \mathbf{I}
\end{array}\right) \quad \text { and } \quad \mathbf{G}^{-1}=\left(\begin{array}{cc}
g \mathbf{I} & \overline{\mathbf{R}} \\
-\mathbf{R} & g \mathbf{I}
\end{array}\right)
$$

where I denotes the identity matrix. The $2 \times 2$ matrix $\mathbf{R}$ in Eq. (7) contains the complete global coupling information. From $\mathbf{G G}^{-1}=\mathbf{I}$ it follows $g^{2}+\operatorname{det} \mathbf{R}=1$, and the quantities $\mathbf{R}$ and $g$ can be computed as [2]

$$
\mathbf{R}=\frac{\mathbf{C}+\overline{\mathbf{B}}}{2\left(\cos \mu_{A}-\cos \mu_{D}\right)}
$$

and

$$
g=\sqrt{1-\frac{\operatorname{det}|\mathbf{C}+\overline{\mathbf{B}}|}{4\left(\cos \mu_{A}-\cos \mu_{D}\right)^{2}}}
$$

The minimum tune approach is [2]

$$
\Delta Q_{\min }=\frac{\sqrt{\operatorname{det}|\mathbf{C}+\overline{\mathbf{B}}|}}{\pi\left(\sin \mu_{A}+\sin \mu_{D}\right)}
$$

and global decoupling amounts to manipulations that result in $\operatorname{det}|\mathbf{C}+\overline{\mathbf{B}}|=0$. Note that the sign of $\operatorname{det}|\mathbf{C}+\overline{\mathbf{B}}|$ is negative on sum resonances and positive on difference resonances [2].

The matrix $\mathbf{R}$ in Eq. (8) is only defined if $\mu_{A} \neq \mu_{D}$. The condition

$$
\mu_{A}=\mu_{D}
$$

can only be obtained in the uncoupled case $\Delta Q_{\text {min }}=0$. In this case $\mathbf{B}=\mathbf{C}=0$ and the matrix $\mathbf{M}$ is already block diagonal, thus $\mathbf{G}=\mathbf{I}, \mathbf{R}=\mathbf{0}$, and $g=1$.

\section{ACKNOWLEDGMENTS}

I am thankful to Zhang Yuan, IHEP, Bejing, for pointing out this problem, and further discussions.

[1] W. Fischer, Phys. Rev. ST Accel. Beams 6, 062801 (2003).

[2] L. Schachinger and R. Talman, "Manual for the Program TEAPOT, Noninteractive fortran Version, Appendix G, 1996. 